\title{
Using social media to educate women and healthcare providers on endometriosis: preliminary results
}

\author{
Márcia Mendonça Carneiro ${ }^{1,2,3}$, Barbara Lopes Farace ${ }^{1,2}$, Luisa Silva de Carvalho Ribeiro ${ }^{1,2}$, Raphaela Cristina \\ Conrado C Silverio ${ }^{1,2}$, Thais Moreira ${ }^{1,2}$, Agnaldo Lopes da Silva Filho ${ }^{1,2}$, Ana Luiza Lunardi Rocha Baroni ${ }^{1,2}$, Márcia \\ Cristina França Ferreira ${ }^{1,2}$ \\ ${ }^{1}$ Department of Gynecology and Obstetrics. School of Medicine of the Federal University of Minas Gerais. Belo \\ Horizonte, Minas Gerais, Brazil \\ ${ }^{2}$ Multidisciplinary Endometriosis and Chronic Pelvic Pain Team. Hospital das Clínicas da UFMG. Belo Horizonte \\ -MG - Brazil \\ ${ }_{3}^{3}$ Multidisciplinary Endometriosis Team. Hospital Biocor and Mater Dei Hospitals - Belo Horizonte -MG - Brazil
}

\begin{abstract}
Objective: This study aimed to evaluate the performance of our Facebook fan page and compare it to similar pages.

Methods: The authors reviewed page-specific metrics provided by Facebook, including number of fans, their age and sex, and country of origin; number of views, likes, and shares tied to our posts. A search for similar fan pages using keyword "endometriosis" found pages in Portuguese, English, and Spanish. They were analyzed for country of origin, authors, number of fans and posts, and were compared to our page.

Results: Our fan page went online on April 2016 and 127 posts have been published up to December 2017. Most of its 1746 fans ( $89 \%$ women; $11 \%$ men) were Brazilians $(n=1677)$. Our audience comprised primarily women aged $25-34(39 \%)$ and $35-44(30 \%)$ years. The most viewed posts discussed thrombosis and oral contraceptives ( $n$ $=11078)$; continuous use of oral contraceptives $(n=6448)$; deep endometriosis $(n=4425)$; intrauterine levonorgestrel system ( $n=4045)$; IVF and endometriosis $(n=2885)$; and endometriosis and cancer $(n=2431)$. Our search found 399 Facebook pages from 44 different countries. The United States $(n=81)$, Brazil $(n=49)$, and the United Kingdom $(n=46)$ topped the list of countries hosting pages, while most of the fan pages were written in English. The majority of the pages were active and $20 \%$ claimed they were based on science. Physicians administered four of the 10 most popular pages. Page heterogeneity prevented comparisons between them.

Conclusion: Men and women have visited our Facebook fan page to obtain information on endometriosis. The pages found in our search were exceedingly heterogeneous and were not compared. Developing pages with sound information is an important step toward educating individuals about endometriosis and helping them make informed choices on available treatments.
\end{abstract}

Keywords: endometriosis, social media, patient education

\section{INTRODUCTION}

Endometriosis is a benign gynecological disorder characterized by the presence and growth of endometrium-like tissue in sites outside the uterine cavity, primarily on the pelvic peritoneum and ovaries (Giudice, 2010; Borghese et al ., 2018). It affects $5-10 \%$ of women of reproductive age and the main clinical features are chronic pelvic pain and infertility (Carneiro et al ., 2010; Borghese et al ., 2018).
Disease hallmarks vary, and there is a lack of pathognomonic symptoms and useful noninvasive clinical tests to diagnose individuals with symptomatic disease. In addition, clinical presentation also varies, with some women experiencing severe symptoms while others remain asymptomatic (Carneiro et al ., 2010; Borghese et al ., 2018). Consequently, delays of five to 11 years have been observed in the diagnosis of individuals with the condition (Ballard et al ., 2006). The need for invasive diagnostic tools, the complexity of clinical presentation, the multiple morphologies of endometriotic lesions, and the lack of well-designed studies with sufficient numbers of patients have hampered research and delayed the diagnosis and introduction of appropriate treatment for the disease (D'Hooghe et al ., 2003; Arruda et al ., 2003).

Although much has been published on diseas awareness from the side of physicians, studies have indicated that women suffering from endometriosis undergo multiple medical evaluations and have their complaints dismissed or not taken seriously, which may contribute to diagnosis delay (Arruda et al ., 2003; Ballard et al ., 2006; Hudelist et al ., 2012). In addition, there is a paucity of published studies on the perceptions the general public has on endometriosis (Shah et al ., 2010). Unfortunately, the quality of information available is often unreliable and leads to unjustified fear and a sense of helplessness (Canis et al ., 2016). Studies have found that women are keen to learn more about endometriosis and its symptoms, and that they preferentially obtain information from schools or the Internet (Shadbolt et al ., 2013). Therefore, delays in diagnosing the condition might be mitigated if the general public were educated about the symptoms of endometriosis (Arruda et al ., 2003; Seear, 2009; Shah et al., 2010; Hudelist et al ., 2012).

Online availability of health information has skyrocketed in recent years. People all over the world use the Internet as their primary source of health information, but unfortunately clear standards are not in place and information is often inaccurate (Hirsch et al ., 2017). Social media has been increasingly used to obtain health information and has certainly changed the way people seek and share information on healthcare, despite the apparent lack of information quality control. Little information is available on the uses, risks, and benefits of using social media to educate the general public, patients, and healthcare professionals (Moorhead et al. , 2013). This study aimed to assess the performance of a Facebook fan page developed by the Endometriosis Multidisciplinary Team of the Federal University of Minas Gerais to communicate reliable information on endometriosis and pelvic pain. An attempt was made at comparing our page to other pages in terms of number of fans, page activity, and post topics. 


\section{MATERIAL AND METHODS}

Facebook was chosen for its status as the most used social media platform (https://br.newsroom.fb.com/company-info/). Our fan page was designed by the Endometriosis Multidisciplinary Team of the Federal University of Minas Gerais (UFMG) to educate visitors on matters related to endometriosis, including infertility. Topics were selected based on the most frequent questions and misconceptions presented by women during consultation. Sometimes topics were chosen based on news stories featuring issues related to endometriosis.

Since there is no defined standard of care or legal guideline on the use of social media in Medicine, we followed the American Congress of Obstetricians and Gynecologists (ACOG) Practice Committee Opinion on Professional Use of Digital and Social Media (ACOG, 2015) and the recommendations of the Brazilian Board of Medicine on safe online communication (CFM - Conselho Federal de Medicina, 2011). When developing content, we took into consideration the Social Media Guidelines and Best Practices published by the Centers for Disease Control and Prevention (CDC, 2012).

In summary, we aimed at keeping the content short and simple with weekly publications. All content was checked for scientific and medical accuracy. The page was closely monitored so that fan questions, comments, and feedback were promptly responded. Page-specific metrics provided by Facebook - number of fans, their age and sex, and country of origin; number of views, likes, and shares of each post - were regularly collected. As a rule, medical consultation was not provided online and visitors were encouraged to read the information provided and take their questions to a physician.

The team responsible for the page was made up of three professors from the Department of Obstetrics and Gynecology of the School of Medicine of the Federal University of Minas Gerais (UFMG) and four medical students supervised by Dr. Carneiro. The page did not accept ads or paid content.

We searched Facebook for fan pages using keyword "endometriosis" and found pages in Portuguese, English, and Spanish. Relevant pages were selected and the following data obtained: country of origin, authors (healthcare provider, medical doctor, or lay people), number of fans, number of posts, and posting frequency. We also tried to compare these pages against ours in terms of number of fans, page activity, and post topics. Additional metrics from the found pages such as fan age, sex, or country of origin were not available to us.

\section{RESULTS}

Our Facebook fan page went online on April 21, 2016, and 127 posts had been published up to December 2017. Most of its 1746 fans ( $89 \%$ women; $11 \%$ men) were Brazilians $(n=1677)$ from the state of Minas Gerais, although individuals from other Brazilian states, the United States, and Europe have visited it. Our audience comprised primarily women aged 25-34 (39\%) and 35-44 (30\%) years, followed by younger females aged $18-24$ years ( $9 \%)$. Men aged 25-35 years accounted for the majority of male fans $(4 \%)$, followed by men aged $35-44$ years (3\%). Most visitors viewed our fan page on cell phones or tablets. The posts with more views discussed the following topics: thrombosis and oral contraceptives $(n=11078)$; continuous use of hormonal contraceptives $(n=6448)$; deep infiltrating endometriosis $(n=4425)$; use of the intrauterine levonorgestrel system ( $n=4045)$; IVF and endometriosis $(n=2885)$; endometriosis and cancer $(n=2431)$; endometriosis and infertility $(n=1299)$; and how to schedule an appointment $(n=1264)$. The numbers keep increasing day after day.

The search for other Facebook pages using keywords "endometriosis" and "endometriose" (Portuguese for endometriosis) found 399 pages from 44 different countries. The United States $(n=81)$, Brazil $(n=49)$, and the United Kingdom $(n=46)$ topped the list of countries hosting pages. Most of the fan pages were written in English $(n=272)$, Spanish $(n=73)$, or Portuguese $(n=51)$. In terms of activity, $311(77.9 \%)$ fan pages were deemed active - at least one post in 2017 - and $20 \%$ claimed they were based on science (their owners included healthcare providers, mainly physicians). A Brazilian fan page from a clinic in São Paulo amassed the greatest number of fans $(59,666)$. Physicians administered four of the 10 most popular pages.

The pages were extremely heterogeneous, and their differences in terms of objectives and content prevented further comparisons. Metrics from each fan page are available only to the administrators of each individual fan page, and therefore were not used for purposes of comparison.

\section{DISCUSSION}

Although our search yielded numerous fan pages on endometriosis, they were hardly comparable. Social media platforms may be a valuable tool to educate individuals about diseases and treatments. Persons with chronic conditions might be particularly engaged for the interest they usually show in learning more about their illness (Antheunis et al ., 2013; Griffiths et al ., 2015; Roberts et al ., 2017). Unfortunately, misinformation about health spreads easily across social media and physicians are often unable to meet all patient education needs (Brown et al ., 2014). Information exchanged via digital means of communication should be monitored for quality and reliability (Tonsaker et al ., 2014).

The majority of our fans (39\%) were young Brazilian women aged 25-34 years. A Brazilian study showed that patient age was inversely related with the time it takes for females to be diagnosed with endometriosis (Arruda et al ., 2003). Therefore, educating women in this age group might shorten the time until diagnosis, improve their quality of life, and decrease healthcare costs (Arruda et al ., 2003; Hudelist et al ., 2012; Seear, 2009). The interest sparked around the condition in social media represents a golden opportunity to educate women on endometriosis, since they prefer to acquire information on the subject from schools and the Internet (Shadbolt et al ., 2013). Unfortunately, we were unable to find studies on the use of social media to educate the public on endometriosis.

Although the majority of our fans were women (89\%), men also showed interest in the published posts. Shah et al . (2010) showed that men and women have limited knowledge of the signs and symptoms of endometriosis, which in turn may contribute to delays in diagnosis. Educating physicians may also decrease delays in diagnosis (Arruda et al ., 2003). Hirsch et al . (2017) published a recent evaluation of information available online on endometriosis. The authors did not find Internet pages that contained high quality, accurate, credible health information about endometriosis. Unfortunately, this may result in patient misinformation, an issue physicians must be aware of.

Despite the recognized role of social media in providing information, healthcare providers and public healthcare institutions are still reluctant to use social media platforms (Antheunis et al ., 2013; Moorhead et al ., 2013). Physicians have not fully adhered to online communication or social media in daily practice (Brown et al ., 2014). However, studies showed that people rely on information available online and many times use the web as a primary source of information (Antheunis et al ., 2013; Griffiths 
et al., 2015). Patients apparently search the Internet not as a substitute for advice from a physician, but as an attempt to gain more knowledge of their condition and of what their physicians told them. This may allow patients to play a more active role in therapy and improve communications with their physicians (Al Ghamdi \& Moussa, 2012; Griffiths et al ., 2015; Roberts et al ., 2017).

Various explanations have been advanced for diagnostic delays, with implications touching physicians and female patients. Women treated for irregular periods might have difficulty distinguishing normal cycles from abnormal cycles, and might therefore take longer to seek help for endometriosis. It has been suggested that diagnostic delays might be reduced if women were informed of what to expect in normal and abnormal menstrual cycles (Seear, 2009).

Strategies to tackle this issue include the development of programs and training courses to improve the diagnostic skills of present and future gynecologists and family physicians. In addition, education initiatives targeting the general population may help to build awareness of endometriosis as an important cause of menstrual pain and subfertility, thereby urging affected women to seek evaluation and treatment. Social media may play a vital role in this setting.

Social media may also enhance patients' access to healthcare information and other educational resources (Moorhead et al ., 2013; Roberts et al ., 2017). Eighty percent of Internet users search specifically for health-related information, and many search for physicians, healthcare providers, and treatment options (Warden, 2017). In 2016, Facebook reported that approximately one billion people had the Facebook app on their smartphones, and that a significant portion of them used the app to search for health-related information.

The posts with more views discussed topics often seen with controversy and fear by the public. The use of oral contraceptives and the alleged associated risk of thrombosis, the risk of cancer in women with endometriosis, along with the potential risk of infertility in women with endometriosis ranked atop the list of post views. This may reflect the debate surrounding these topics and the need for reliable sources of information to deal with questions left unanswered after consultation with a physician. Social media is a golden opportunity to educate the public, dissipate myths, and provide patients with sound information to influence their decisions (Moorhead et al ., 2013; Roberts et al ., 2017). Unfortunately, misinformation about health spreads easily across social media and influences patient decisions.

In conclusion, we have developed a Facebook fan page for men and women of reproductive age seeking information on endometriosis. Preliminary findings indicated that our page on endometriosis has attracted the attention of males and females. Although many fan pages on endometriosis are available, they are quite heterogeneous and the quality of published information cannot be easily assessed. Developing pages with sound information is an important step toward educating men and women about endometriosis and helping them make informed choices on fertility and available treatments. Physicians and healthcare professionals should seize the opportunity and use social media to promote patient healthcare and education.

\section{CONFLICT OF INTEREST}

The authors have no conflict of interest to declare.

\section{Corresponding author:}

Márcia Mendonça Carneiro

Department of Obstetrics and Gynecology

Universidade Federal de Minas Gerais

Belo Horizonte, Minas Gerais, Brazil.

E-mail: marciamc.ufmg@gmail.com

\section{REFERENCES}

ACOG. American College of Obstetricians and Gynecologists. Committee on Professional Liability. Professional use of digital and social media. Committee Opinion No. 622. Obstet Gynecol. 2015;125:516-20. PMID: 25611641 DOI: 10.1097/01.AOG.0000460783.32467.bf

Al Ghamdi KM, Moussa NA. Internet use by the public to search for health-related information. Int J Med Inform. 2012;81:363-73. PMID: 22217800 DOI: 10.1016/j.ijmedinf.2011.12.004

Antheunis ML, Tates K, Nieboer TE. Patients' and health professionals' use of social media in health care: motives, barriers and expectations. Patient Educ Couns. 2013;92:42631. PMID: 23899831 DOI: 10.1016/j.pec.2013.06.020

Arruda MS, Petta CA, Abrão MS, Benetti-Pinto CL. Time elapsed from onset of symptoms to diagnosis of endometriosis in a cohort study of Brazilian women. Hum Reprod. 2003;18:756-9. PMID: 12660267 DOI: 10.1093/humrep/deg136

Ballard K, Lowton K, Wright J. What's the delay? A qualitative study of women's experiences of reaching a diagnosis of endometriosis. Fertil Steril. 2006;86:1296-301. PMID: 17070183 DOI: $10.1016 /$ j.fertnstert.2006.04.054

Borghese B, Santulli P, Marcellin L, Chapron C. Definition, description, clinicopathological features, pathogenesis and natural history of endometriosis: CNGOF-HAS Endometriosis Guidelines. Gynecol Obstet Fertil Senol. 2018;46:15667. PMID: 29540335 DOI: 10.1016/j.gofs.2018.02.017

Brown J, Ryan C, Harris A. How doctors view and use social media: a national survey. J Med Internet Res. 2014;16:e267. PMID: 25470407 DOI: 10.2196/jmir.3589

Canis M, Curinier S, Campagne-Loiseau S, Kaemerlen Rabischong AG, Rabischong B, Pouly JL, Grémeau AS, Botchorishvili R, Bourdel N. Information to patients in endometriosis: We must stop the frightening machine! Gynecol Obstet Fertil. 2016;44:618-9. PMID: 27765429 DOI: 10.1016/j. gyobfe.2016.09.009

Carneiro MM, Filogonio IDS, Costa LMP, Avila I, Ferreira MC. Accuracy of clinical signs and symptoms in the diagnosis of endometriosis. J Endometr Pelvic Pain Disord. 2010;2:63-70.

CDC - Center for Disease Control and Prevention. Social Media Guidelines and Best Practices Centers; 2012. Available at: https://www.cdc.gov/SocialMedia/Tools/guidelines/pdf/FacebookGuidelines.pdf

CFM - Conselho Federal de Medicina. Manual de publicidade médica: resolução CFM no 1.974/11 / Conselho Federal de Medicina; Comissão Nacional de Divulgação de Assuntos Médicos. Brasília: CFM; 2011. Available at: https://portal. cfm.org.br/publicidademedica/pubpropaganda4.html 
D'Hooghe TM, Debrock S, Mueleman C, Hill JA, Mwenda JM. Future directions in endometriosis research. Obstet Gynecol Clin North Am. 2003;30:221-44. PMID: 12699268 DOI: $10.1016 / S 0889-8545(02) 00063-3$

Griffiths F, Dobermann T, Cave JA, Thorogood M, Johnson S, Salamatian K, Gomez Olive FX, Goudge J. The Impact of Online Social Networks on Health and Health Systems: A Scoping Review and Case Studies. Policy Internet. 2015;7:473-96. PMID: 27134699 DOI: 10.1002/poi3.97

Hirsch M, Aggarwal S, Barker C, Davis CJ, Duffy JMN. Googling endometriosis: a systematic review of information available on the Internet. Am J Obstet Gynecol. 2017;216:451-8.e1. PMID: 27840143 DOI: 10.1016/j.ajog.2016.11.1007

Hudelist G, Fritzer N, Thomas A, Niehues C, Oppelt P, Haas D, Tammaa A, Salzer H. Diagnostic delay for endometriosis in Austria and Germany: causes and possible consequences. Hum Reprod. 2012;27:3412-6. PMID: 22990516 DOI: 10.1093/humrep/des316

Moorhead SA, Hazlett DE, Harrison L, Carroll JK, Irwin $A$, Hoving $C$. A new dimension of health care: systematic review of the uses, benefits, and limitations of social media for health communication. J Med Internet Res. 2013;23:15:e85. PMID: 23615206 DOI: 10.2196/ jmir.1933
Roberts M, Callahan L, O'Leary C. Social Media: A Path to Health Literacy. Stud Health Technol Inform. 2017;240:464-75. PMID: 28972534 DOI: 10.3233/978-161499-790-0-464

Shadbolt NA, Parker MA, Orthia LA. Communicating endometriosis with young women to decrease diagnosis time. Health Promot J Austr. 2013;24:151-4. PMID: 24168743 DOI: $10.1071 / \mathrm{HE} 12915$

Shah DK, Moravek MB, Vahratian A, Dalton VK, Lebovic DI. Public perceptions of endometriosis: perspectives from both genders. Acta Obstet Gynecol Scand. 2010;89:64650. PMID: 20235893 DOI: 10.3109/00016341003657900

Seear K. The etiquette of endometriosis: stigmatisation, menstrual concealment and the diagnostic delay. Soc Sci Med. 2009;69:1220-7. PMID: 19699572 DOI: 10.1016/j.socscimed.2009.07.023

Tonsaker T, Bartlett G, Trpkov C. Health information on the Internet: gold mine or minefield? Can Fam Physician. 2014;60:407-8. PMID: 24828994

Warden C. 30 Facts \& Statistics On Social Media And Healthcare. Mt. Pleasant, SC: Referral MD - Referral Management Software; 2017. Available at: https://getreferralmd.com/2017/01/30-facts-statistics-on-social-mediaand-healthcare/ 